\title{
Phototherapy with probiotics supplementation therapy and phototherapy alone in neonates with jaundice: A randomized clinical trial
}

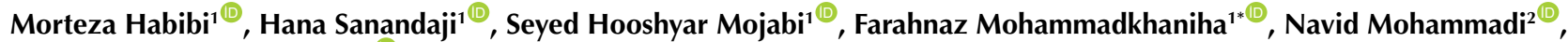 \\ Marzieh Mohammadhoseini ${ }^{10}$
}

${ }^{1}$ Clinical Research Development Unit, Kowsar Hospital, Qazvin University of Medical Sciences, Qazvin, Iran

${ }^{2}$ Children Growth Research Center, Research Institute for Prevention of Non-Communicable Diseases, Qazvin University of Medical

Sciences, Qazvin, Iran; Researcher, and Canada Optimax Access Consultation, Ottawa, Canada

\section{*Correspondence to Farahnaz Mohammadkhaniha, Email: \\ f.mohammdkhaniha@qums. ac.ir}

Received 9 Apr. 2021 Accepted 3 July 2021 Published online 30 July 2021

Keywords: Probiotic, Neonatal, Total bilirubin, Direct bilirubin, Hospitalization days

\begin{abstract}
Introduction: Probiotics are non-pathogenic strains of organisms. These strains alter the microbial ecology of the gut and improve bowel function.

Objectives: In this study, the researchers aimed to evaluate the effect of probiotics on reducing the duration of treatment for neonatal jaundice and the rate of readmission due to recurrence.

Patients and Methods: In this randomized clinical trial, 88 neonates with jaundice hospitalized for phototherapy in Kowsar hospital from April 2020 to February 2021 were studied. Eligible neonates were randomly divided into probiotic and placebo groups. Both groups received standard conventional phototherapy and the intervention group received 5 drops of probiotic until hospital discharge. The outcome variables were bilirubin, hospitalization days, and readmission.

Results: The probiotic group had a significantly lower hospitalization stay in comparison to the placebo group. The hospitalization of the probiotic group was $2.43 \pm 0.82$ days, while the hospitalization of the placebo group was $5.18 \pm 2.05$ days.

Conclusion: Using oral probiotics in neonates with jaundice has a significant effect on reducing hospitalization days and readmission. Further studies are needed with longer time follow-up.

Trial Registration: The trial protocol was approved in the Iranian Registry of Clinical Trial website (identifier: IRCT20191022045194N1, https://en.irct.ir/trial/43675, ethical code (\#IR.QUMS.REC.1398.041).
\end{abstract}

\section{Introduction}

Neonatal hyperbilirubinemia is a clinical disorder in which several factors cause the inability of the liver to produce, combine, absorb, and excrete bilirubin which manifests as neonatal jaundice (1). Jaundice appears during the early neonatal period, depending on etiology. Jaundice is the result of indirect bilirubin deposition on the skin which causes the skin to turn yellow or light orange. In obstructive jaundice (direct bilirubin), the skin becomes greenish or muddy yellow. Phototherapy, intravenous immunoglobulin (IVIG), metalloporphyrin, and blood transfusion are used to treat neonatal jaundice (2). Phototherapy can change the structure of bilirubin and increase its excretion. This is currently the standard treatment for jaundice (3). The phototherapy device can have shortand long-term side effects for the baby one of which is damage to the microbial flora of the intestine. Therefore, some clinicians tend to

\section{Key point}

In the treatment of neonatal jaundice, the use of probiotics can significantly reduce the number of hospitalization days as well as readmission due to the recurrence of the disease.

use probiotics to treat neonatal jaundice (4).

Probiotics improve people's health (5). Bifidobacterium and Lactobacillus are the organisms which exist in probiotics (6). Probiotics can reduce the risk and severity of jaundice. In addition, their ease of use, low cost, and availability make them suitable for treating jaundice. Therefore, this study aimed to evaluate the effects of probiotics on reducing jaundice as well as the number of hospitalization days.

\section{Objectives}

Long-term treatment with the phototherapy device causes many complications for the

\footnotetext{
Copyright (C) 2022 The Author(s); Published by Nickan Research Institute. This is an open-access article distributed under the terms of the Creative Commons Attribution License (http://creativecommons.org/licenses/by/4.0), which permits unrestricted use, distribution, and reproduction in any medium, provided the original work is properly cited.
} 
baby. Therefore, to prevent possible complications, the researchers decided to use oral probiotics to reduce the length of hospital stay.

\section{Patients and Methods}

\section{Study design}

This clinical trial was conducted on 88 infants with jaundice in the Neonatal Unit of Kowsar Educational, Research, and Medical Center of Qazvin from 2020 to 2021. The study was conducted on 88 infants with neonatal hyperbilirubinemia selected through convenience sampling.

Two combined methods were used for selecting the case and control groups. First, the infants were visited by a neonatologist and their health was confirmed. Based on a random system, the infants were divided into two groups to carry out the project. The subjects were assigned to probiotic $(\mathrm{n}=44)$ and control groups $(\mathrm{n}=44)$ by random allocation. The inclusion criteria were birth weight of more than 2000 g, gestational age under 35 weeks (fertilization age was 27 weeks and did not exceed 35 weeks), having no diseases (healthy babies who had no problems or illnesses in the first week of life), and Iranian nationality. The exclusion criteria were the parents' dissatisfaction with continuing to participate in the research, the neonates' death or critical condition, the neonates' discharge before the end of the study, congenital anomalies, sepsis, thrombocytopenia, diastolic pressure greater than $60 \mathrm{~mm}$ $\mathrm{Hg}$, shock, hydrops fetalis, inflammation, seizures, and hemolytic diseases. The researchers first explained the goals and methods of the research to the infants' parents and after receiving their informed consent, they started the intervention. The data collection tools included a demographic record, a probiotic checklist, and a neonatal bilirubin checklist.

In the placebo group, based on bilirubin levels, phototherapy and distilled water were administered. In this group, the infants were given a five $\mathrm{ml}$ distilled water pack (Shahid Ghazizadeh Pharmaceutical Co.). In the probiotic group, in addition to phototherapy, five drops of oral probiotic (Pedilact) were given to the infants twice a day (Zist Takhmir Co.). Then, the bilirubin changes and the number of hospitalization days were measured. On the day of discharge, the total and direct bilirubin levels were measured in the two groups and the number of hospitalization days for phototherapy treatment was also recorded.

\section{Data analysis}

The data collected in this study were analyzed by SPSS software (version 21). An independent T-test and ANOVA were used to compare the relationship between probiotics and bilirubin results. A p-value of less than 0.05 was considered a significant level. The data were analyzed using 2-way ANOVA for repeated measures between groups and an independent t-test for continuous variables. P-values of less than 0.05 were considered significant.

\section{Results}

The CONSORT diagram for the study is depicted in Figure 1. Eighty-eight neonates were in the study including 44 neonates in the probiotic group and 44 preterm infants in the placebo group. The probiotic and placebo groups were comparable in age, gender, and bilirubin. They received the same appropriate management except for the use of probiotics or placebo. Table 1 summarizes the demographic and clinical characteristics as well as the neonatal outcomes of the probiotic group versus the placebo control group. The average age of the participants in the probiotic group was $33.05 \pm 2.2$ weeks and the majority of the neonates were male (50, 56.8\%).

Using repeated measurement and independent t-test, the study showed non-significant differences between the two groups concerning bilirubin on the hospitalization and discharge days. In addition, the median total and direct bilirubin levels were comparable in both groups $(P<0.001$; Table 1). The present study investigated the effects of probiotics and non-probiotics on the neonates' bilirubin levels and hospitalization days. The results showed significant decreases in the total and direct bilirubin levels of the two groups. The neonatal total bilirubin levels in the probiotic group on the hospitalization and discharge days were $14.55 \mathrm{mg} / \mathrm{dL}$ and $10 \mathrm{mg} / \mathrm{dL}$, respectively, whereas in the placebo group, they were $14.07 \mathrm{mg} / \mathrm{dL}$ and $10 \mathrm{mg} / \mathrm{dL}$, respectively. In addition, the neonatal total bilirubin levels in the probiotic group on the hospitalization and discharge days were $0.7 \mathrm{mg} / \mathrm{dL}$ and $0.51 \mathrm{mg} / \mathrm{dL}$, respectively, while in the placebo group, they were $0.5 \mathrm{mg} / \mathrm{dL}$ and $0.46 \mathrm{mg} /$ $\mathrm{dL}$ respectively. The total and direct bilirubin levels of the two groups were reduced almost equally in the two groups. The mean numbers of hospitalization days in the probiotic and placebo groups were $2.43 \pm 0.82$ and $5.18 \pm 2.5$ days, respectively. Furthermore, in the placebo group, eight infants were hospitalized again after discharge due to increased bilirubin. The total bilirubin and direct bilirubin levels at the time of readmission were $15.42 \pm 2.13 \mathrm{mg} / \mathrm{dL}$ and $0.48 \pm 0.09 \mathrm{mg} / \mathrm{dL}$, respectively.

\section{Discussion}

Hyperbilirubinemia treatment aimed to prevent the indirect bilirubin levels from reaching the point at which neurotoxicity, deafness, and kernicterus may occur. Intensive and single Phototherapy is the treatment of choice for infant hyperbilirubinemia and was administered equally for the infants in both groups. A variety of drugs prescribed such as phenobarbital and clofibrate have been proposed for reducing hospitalization duration. The purpose of probiotics is to increase the number of beneficial bacteria in the bowels. Recently, probiotics are useful for treating gastroenteritis by slowing bacterial growth. The mean levels of total and direct bilirubin were compared in each group. The bilirubin level decreased significantly compared to the day of hospitalization. However, this difference was not significant between 


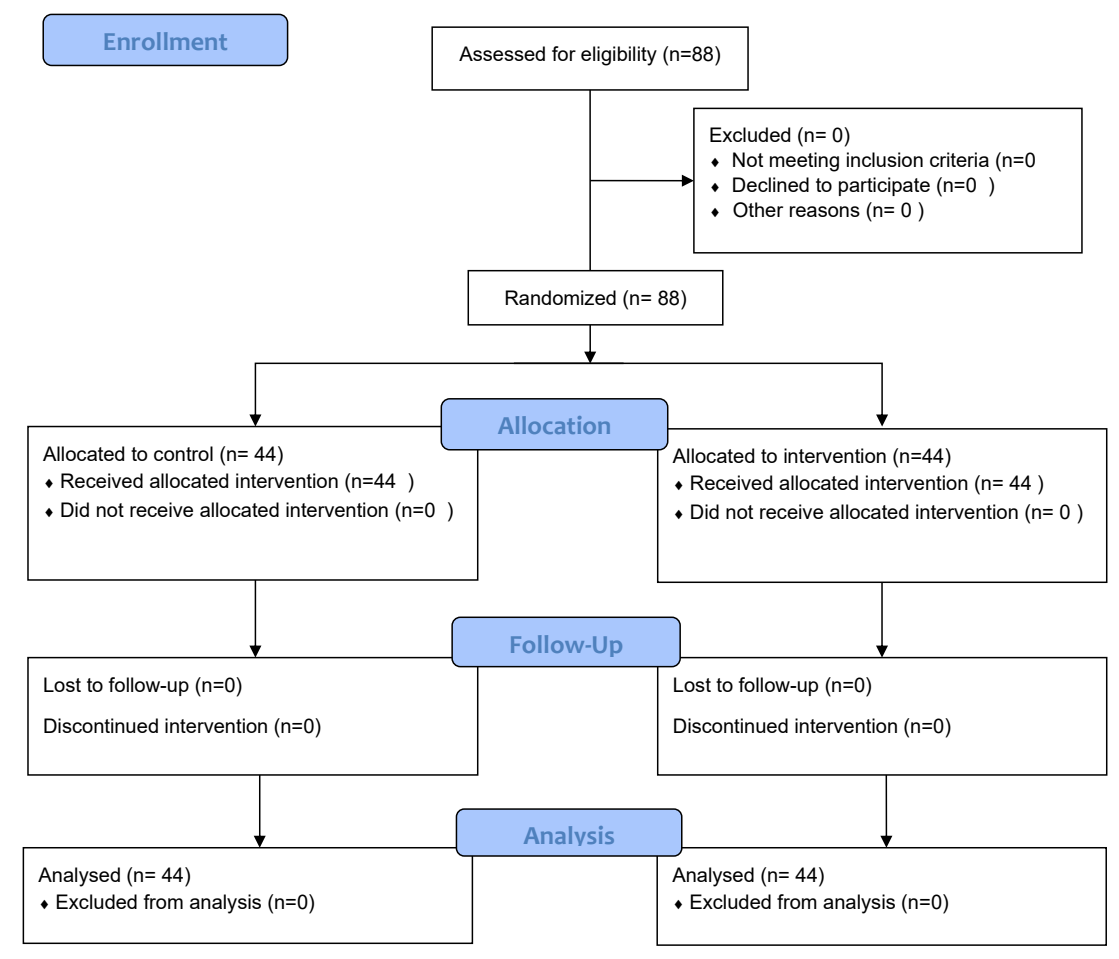

Figure 1. The flowchart of the study.

the two groups (Table 1). Nevertheless, there was a significant difference between the two groups regarding hospitalization $(P<0.001$; Table 1$)$. This means that probiotics can lower a baby's bilirubin in less time. In addition to reducing the hospitalization days, this can also reduce the costs of the health care system. The results of the study of Demirel et al showed that using probiotics reduced the hospitalization days which is consistent with those of the present study (4). According to research by Mutlu et al, probiotic support initiated immediately after birth has a positive effect on bilirubin metabolism and may reduce the risk of jaundice (7). The study of Liu et al also demonstrated Probiotics lowered the serum bilirubin levels rapidly, safely, and significantly, and accelerated jaundice fading as well. Probiotic therapy is worthy of application in clinical practice (8). Moreover, Chen et al. Found in a meta-analysis of 13 clinical trials involving 1067 infants that probiotics supplementation therapy is an effective and safe treatment for pathological neonatal jaundice (9). In a systematic review, Deshmukh et al reviewed nine articles on 2040 infants. They found that probiotic supplementation may reduce the duration of phototherapy in infants with jaundice (10). Therefore, the results of these studies are consistent with those of the

Table 1. Demographic characteristics and outcomes of the patients in probiotic and placebo group $(\mathrm{N}=88)$

\begin{tabular}{|c|c|c|c|}
\hline & Intervention group $(n=40)$ & Control group $(n=40)$ & $P$ value* \\
\hline Gender, No. (\%) & & & 0.671 \\
\hline Female & $24(54.5)$ & $26(59.1)$ & \\
\hline Male & $20(45.5)$ & $18(40.9)$ & \\
\hline Gestational age (wk) & & & 0.959 \\
\hline$<37$ & $4(9.1)$ & $6(13.6)$ & \\
\hline$>37$ & $40(90.9)$ & $38(86.4)$ & \\
\hline Birth weight (g) & & & 0.999 \\
\hline$<2500$ & $4(9.1)$ & $6(13.6)$ & \\
\hline$>2500$ & $40(90.9)$ & $38(86.4)$ & \\
\hline Base total bilirubin $(\mathrm{mg} / \mathrm{dL})$, mean $\pm \mathrm{SD}$ & $14.55 \pm 3.36$ & $14.07 \pm 2.97$ & 0.478 \\
\hline Base direct bilirubin $(\mathrm{mg} / \mathrm{dL})$, mean $\pm \mathrm{SD}$ & $0.7 \pm 1.26$ & $0.5 \pm 0.11$ & 0.327 \\
\hline Discharge total bilirubin $(\mathrm{mg} / \mathrm{dL})$, mean $\pm \mathrm{SD}$ & $10 \pm 2.06$ & $10 \pm 1.49$ & 0.985 \\
\hline Discharge direct bilirubin (mg/dL) & $0.51 \pm 0.39$ & $0.46 \pm 0.10$ & 0.435 \\
\hline Hospitalization (day) & $2.43 \pm 0.82$ & $5.18 \pm 2.5$ & 0.001 \\
\hline
\end{tabular}


present study.

In addition, In the present study in the probiotic group, none of the infants was re-hospitalized due to recurrence of jaundice, while in the control group, eight infants in the placebo group were readmitted with high bilirubin levels. The aim of physicians is to reduce the recurrence of jaundice in infants. The authors suggest that additional studies be conducted to further investigate the effects of probiotics on reducing hospitalization days and changing bilirubin levels.

\section{Conclusion}

As a result of all these efforts, it is important for physicians that no recurrence of jaundice be observed in infants. Therefore, the authors suggest that additional studies be conducted to further investigate further aspects of the probiotic effect on reducing hospitalization days and changes in bilirubin levels.

\section{Limitations of the study}

The limitation of the current study was the decrease in births in recent years. Consequently, sample collection took a lot of time.

\section{Acknowledgments}

The authors would like to express their gratitude to the Center for Development of Clinical Research of Kowsar Hospital.

\section{Authors' contribution}

$\mathrm{MH}, \mathrm{HS}, \mathrm{FM}$, and SHM were the main researchers of the study. MM participated in the sample collection. NM performed the statistical analysis. All authors participated in the final draft of the manuscript, revised the manuscript, and critically evaluated the intellectual material. All authors have read and verified the content of the manuscript and verified the accuracy or integrity of each part of the study.

\section{Conflicts of interest}

The authors declare that they have no competing interests.

\section{Ethical issues}

All the principles of the Helsinki Declaration were observed in this research and it was approved by the Ethics Committee of Qazvin University of Medical Sciences (\# IR.QUMS.REC.1398.041). The trial protocol was approved by the Iranian Registry of Clinical Trials (identifier: IRCT20191022045194N1, https://en.irct.ir). The neonates in the study were hospitalized due to neonatal jaundice.
These conscious infants were in the phototherapy device throughout the treatment except for the time of feeding and changing diapers. Before the study, conscious infants were obtained from their parents. Moreover, ethical issues (including plagiarism, data fabrication, double publication) have been completely observed by the authors. This study was extracted from the dissertation of a pediatric assistant at Qazvin University (dissertation \#659).

\section{Funding/support}

This work supported by deputy research and technology of Qazvin University of Medical Sciences in Iran( proposal code \# 14003738).

\section{References}

1. Bhutani VK, Stark AR, Lazzeroni LC, Poland R, Gourley GR, Kazmierczak S, et al. Predischarge screening for severe neonatal hyperbilirubinemia identifies infants who need phototherapy. J Pediatr. 2013;162:477-482.e1. doi: 10.1016/j. jpeds.2012.08.022.

2. Carter RG, Feigelman S. The Fetus and the Neonatal Infant. In: Kliegman RM, St. Geme J, eds. Nelson Textbook of Pediatrics. 21st ed. Philadelphia: Elsevier; 2020. p. 954-961

3. Faulhaber FRS, Procianoy RS, Silveira RC. Side Effects of Phototherapy on Neonates. Am J Perinatol. 2019;36:252-257. doi: 10.1055/s-0038-1667379.

4. Demirel G, Celik IH, Erdeve O, Dilmen U. Impact of probiotics on the course of indirect hyperbilirubinemia and phototherapy duration in very low birth weight infants. J Matern Fetal Neonatal Med. 2013;26:215-8. doi: 10.3109/14767058.2012.725115.

5. Gill H, Prasad J. Probiotics, immunomodulation, and health benefits. Adv Exp Med Biol. 2008;606:423-54. doi: 10.1007/978-0-387-74087-4_17.

6. McNulty NP, Yatsunenko T, Hsiao A, Faith JJ, Muegge BD, Goodman AL, et al. The impact of a consortium of fermented milk strains on the gut microbiome of gnotobiotic mice and monozygotic twins. Sci Transl Med. 2011;3:106ra106. doi: 10.1126/scitranslmed.3002701.

7. Mutlu M, Irmak E, Aslan Y, Kader S. Effects of Lactobacillus rhamnosus GG as a probiotic on neonatal hyperbilirubinemia. Turk J Pediatr. 2018;60:482-7. doi: 10.24953/ turkjped.2018.05.003.

8. Liu W, Liu H, Wang T, Tang X. Therapeutic effects of probiotics on neonatal jaundice. Pak J Med Sci. 2015;31:1172-5. doi: 10.12669/pjms.315.7921

9. Chen Z, Zhang L, Zeng L, Yang X, Jiang L, Gui G, et al. Probiotics Supplementation Therapy for Pathological Neonatal Jaundice: A Systematic Review and Meta-Analysis. Front Pharmacol. 2017;8:432. doi: 10.3389/fphar.2017.00432.

10. Deshmukh J, Deshmukh M, Patole S. Probiotics for the management of neonatal hyperbilirubinemia: a systematic review of randomized controlled trials. J Matern Fetal Neonatal Med. 2019;32:154-63. doi: 10.1080/14767058.2017.1369520. 